\title{
Studies of brain fatigue detection method based on second-order blind identification
}

\author{
Wang ping, Mu Zhendong ${ }^{1}$ \\ College of Information Engineering, Jiangxi College of Technology, Jiangxi Nanchang 330098
}

Keywords: Fatigue detection; EEG; Second-order blind identification

\begin{abstract}
Brain fatigue detection can make people avoid fatigue on the human body caused by injury, second-order blind identification methods have been successfully used in EEG analysis, because no supervised learning, feature extraction speed is second-order blind identification method is faster, in this paper, the use of second-order blind identification method of the three brain states feature extraction, classification by calculation, the results show a very good second-order blind identification can identify these three states
\end{abstract}

\section{Introduction}

Today's fast-paced life of the community, often working life will make people physically and mentally exhausted, so there is a term "sub" in medicine, that the body of long-term state of tension, causing problems on health. Such as accountants appear on news reports, programmers sudden death, sudden death game players and so on, although the causes of these tragedies could be the cause of these diseases the human body, but long-term brain fatigue can not shirk responsibility. Many people are disorders that can be detected, especially in some chronic diseases, such as by physical characteristics of blood pressure, pulse, etc., fatigue, which affect a new feature of modern health can detect it? Detection by what methods? This is a question many scholars concern.

In-depth research and development of brain wave study brings to this new direction, when the brain of a state change occurred when the results show that these states will bring about changes in brain waves, so the time when the brain fatigue, will produce the appropriate corresponding brain wave analysis of brain waves can be detected very good fatigue of the brain, for example, Beijing Institute of Technology research on driver fatigue, and they passed on the brain wave frequency distribution analysis section, eventually detected brainwave frequencies are different characteristics of human fatigue state, second-order blind identification is a blind source analysis method, this method can achieve a good EEG feature extraction, such as [1 on the use of two Busy order to achieve a brainwave identification method of feature extraction.

In this paper, the use of second-order blind identification method, the EEG under three states were analyzed by calculating the characteristics of brain waves, established state - correspondence between features, and in order to achieve the brain fatigue were detected by brain waves. In the EEG signal input sample test results show that the electrical signals can achieve a good sample of the brain are classified under different conditions, which explain the use of second-order blind identification method can achieve brain fatigue testing.

1 * Corresponding Author: $418623577 @$ qq.com 


\section{Method}

So that $\mathrm{x}(\mathrm{t})$ of the $\mathrm{n}$ column vectors corresponding to the $\mathrm{n}$ successive time EEG sensors, the $\mathrm{xi}(\mathrm{t})$ corresponding to the $\mathrm{i}$-th EEG sensors. Each $\mathrm{xi}(\mathrm{t})$ can be regarded as $\mathrm{n}$ source si (t) is the instantaneous linear mixing, the mixing matrix $\mathrm{A}$, then

$x(t)=A s(t)$

SOBI sensor measurements obtained using only EEG $x$ (t), A-1 to give approximately decomposition matrix $\mathrm{W}$, such that

$\hat{s}(t)=W x(t)$

$\mathrm{Wx}(\mathrm{t})$ is the recovered Continuous-time source signal.

SOBI algorithm has two steps: First, the sensor signal of zero mean, the following formula:

$y(t)=B(x(t)-\langle x(t)\rangle)$

Pointed brackets indicate the average time, so the mean y is zero. The value y of matrix B such that the correlation matrix, $\mathrm{A}$ is a unit matrix, and its value is given by the following formula $B=\operatorname{diag}\left(\lambda_{i}^{-1 / 2}\right) U^{T}$

Where $\lambda \mathrm{i}$ is a characteristic correlation matrix $\left\langle(x(t)-\langle x(t)\rangle)(x(t)-\langle x(t)\rangle)^{T}\right\rangle$, and the columns of $\mathrm{U}$ are the eigenvectors corresponding to its

The second step, construct a set of diagonal matrices: selecting a set of time delay $\tau \mathrm{s}$, calculates a signal $\mathrm{y}(\mathrm{t})$ and its time delayed signal $\mathrm{y}(\mathrm{t}+\tau)$ is symmetric correlation matrix:

$R_{\tau}=\operatorname{sym}\left(\left\langle y(t) y(t+\tau)^{T}\right\rangle\right)$

and then

$\operatorname{sym}(M)=\left(M+M^{T}\right) / 2$

After $\mathrm{R} \tau$ calculations, and then carried on $\mathrm{R} \tau$ diagonalization: by rotation matrix $\mathrm{V}$, using the iterative method, making

$\sum_{\tau} \sum_{i \neq j}\left(V^{T} R_{\tau} V\right)_{i j}^{2}$

Estimated local minimum value, the separating matrix

$W=V^{T} B$

Where $\mathrm{W}$ is the EEG feature library of the state.

\section{Results}

Fatigue and no clear dividing line, but for extreme cases, but also can distinguish, for example, awake, tired state and began to doze off state, which is three states are distinguished, in order to verify that the second order blind identification method feasible, we were collected EEG these three states, and feature extraction, sampling EEG flat rate is $128 \mathrm{~Hz}, 1 \sim 49 \mathrm{~Hz}$ band-pass filtering. 
Herein EEG from 10 subjects, EEG acquisition experiment once a day, every experiment lasted 15 minutes, collecting a total of 10 days for the larger drift EEG, we use scan4.3 delete, so EEG was original database, the original EEG database which is made up of several consecutive EEG components.

When a 3-second intervals interception EEG, we can build the same length EEG group, in order to carry out unified computing, 10 people had opted for 400 as the raw EEG data segment, so the composition of the raw data $10 *$ raw data $384 * 400$.

400 samples for each subject, we chose 200 as the study sample, 200 as a test sample, which two hundred test samples awake 80, overworked state 50, 70 state of drowsiness. $200 * 384$ is the second order so busy recognition input data, after the article describes the method to calculate, 200 test samples for the three fatigue correct classification results shown in Figure 1.

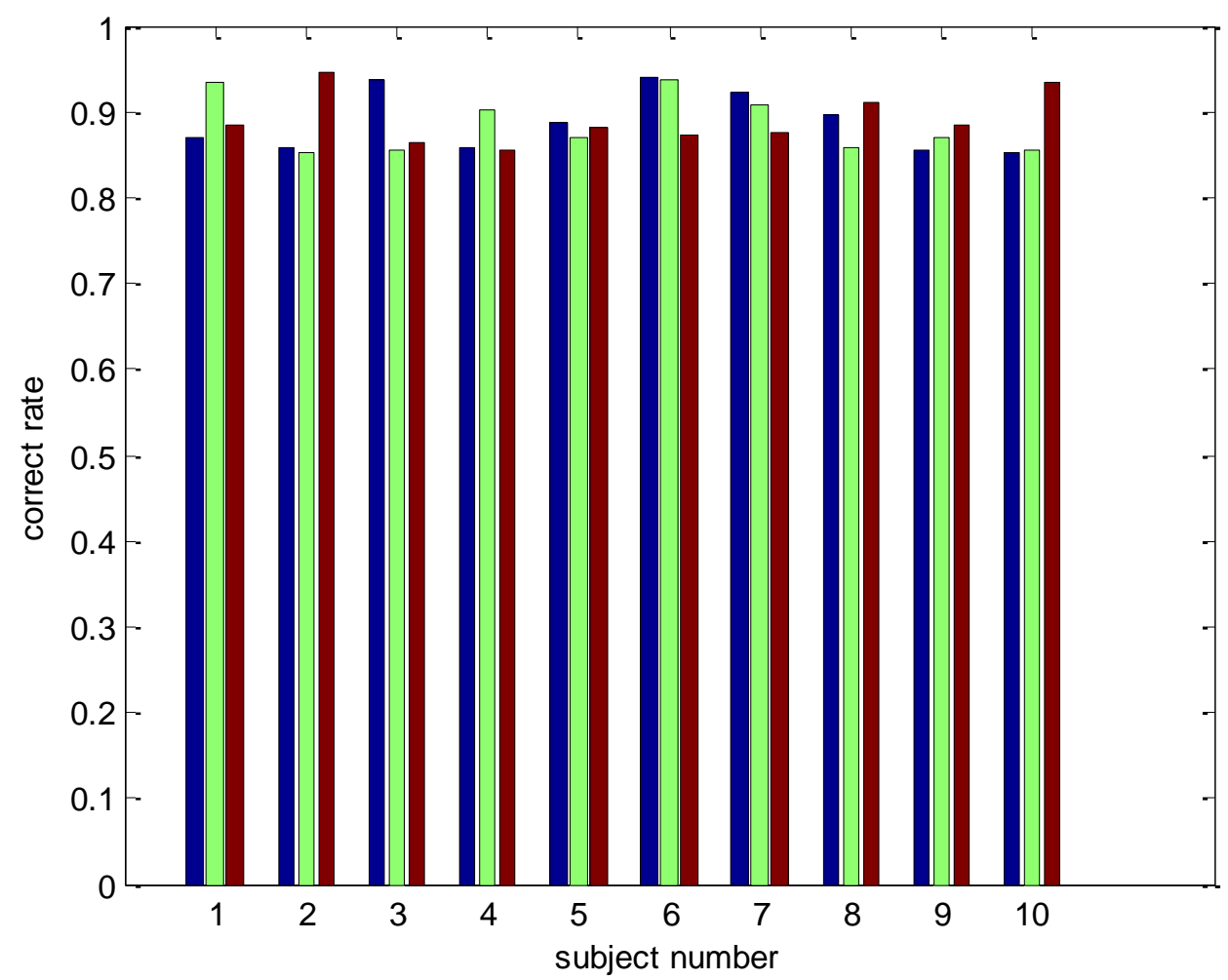

Figure. 1 Correct rate of ten subject

Figure 1 is a ten subjects for three states of classification accuracy, in which the $\mathrm{x}$-axis is the subject number, y-axis is the accuracy, Figure 1 shows that for ten subjects, accurately determine the status of the subject were more than $80 \%$, indicating that the method can determine the degree of fatigue of the subject.

In order to be able to quickly classify calculations, we calculated the impact of different lengths for the accuracy of the data, we calculated the one second, two seconds, three seconds, four seconds and 5 seconds of data is calculated, the accuracy rate was as follows 


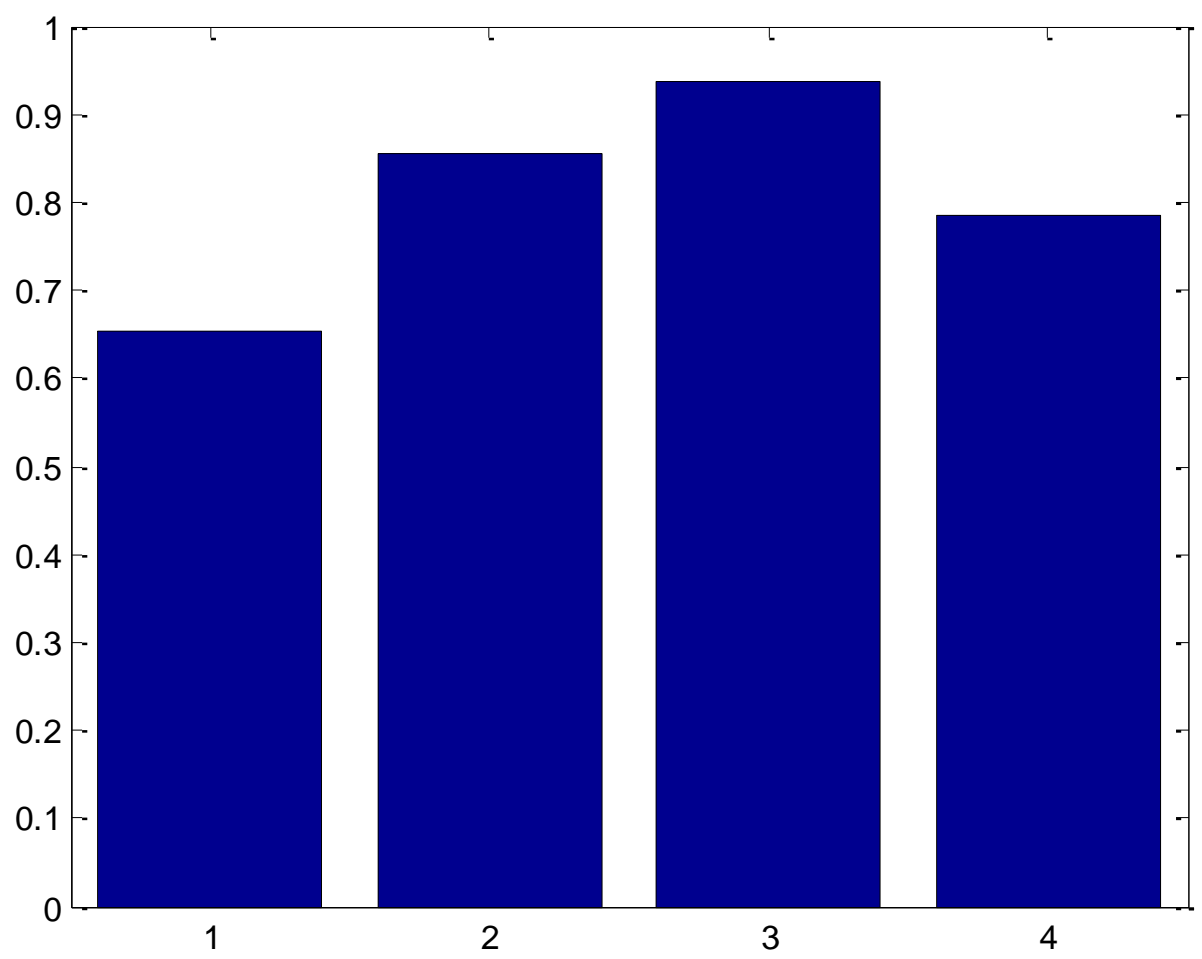

Figure. 2 Effects of different lengths of time for the accuracy

Figure 2 shows the effect of different lengths of time the classification accuracy, in which the $\mathrm{x}$-axis is the length of time, namely, one second, two seconds, three seconds, and four seconds, $y$-axis represents the length of time such a classification accuracy. Figure 2 showed three seconds when the highest average classification accuracy, the accuracy of one second lowest, showing the length of time to affect the classification accuracy.

\section{Acknowledgements}

This work was financially supported by project of Technology Department of Jiangxi Province [No 20143BBM26048] and project of Jiangxi University of Technology [No. xtcx201312].

\section{References}

[1]g A C, Sutherland M T, McKinney C J. Validation of SOBI components from high-density EEG[J]. NeuroImage, 2005, 25(2): 539-553.

[2] Joyce C A, Gorodnitsky I F, Kutas M. Automatic removal of eye movement and blink artifacts from EEG data using blind component separation[J]. Psychophysiology, 2004, 41(2): 313-325. 